\title{
Non-marginally bound inhomogeneous dust collapse in higher dimensional space-time
}

\author{
S.G. Ghosh \\ Department of Mathematics, Science College, Congress Nagar, Nagpur - 440 012, India \\ A. Banerjeef \\ Department of Physics, Jadavpur University, Calcutta 700 032, India
}

(Dated: December 4, 2018)

\begin{abstract}
We investigate the occurrence and nature of a naked singularity in the gravitational collapse of an inhomogeneous dust cloud described by a self-similar higher dimensional Tolman-Bondi space-time. Bound, marginally bound and unbound space-times are analyzed. The degree of inhomogeneity of the collapsing matter necessary to form a naked singularity is given.

PACS numbers: 04.50.+h, 04.20.Dw, 04.70.Bw, 04.20.Jb
\end{abstract}

\section{INTRODUCTION}

The Cosmic Censorship conjecture (CCC) of Penrose [1] states that in generic situation all singularities arising from regular initial data are clothed by event horizon and hence invisible to distant observers. According to strong version of the conjecture, such singularities are invisible to all observers, i.e., there occur no naked singularities for any observer. The conjecture plays a fundamental role in the theory of black holes. Despite the flurry of activity over years, the validity of this conjecture is still an open question (see, e.g., [2] for reviews on conjecture). However, there exist many exact solutions of Einstein equations where naked singularities do arise, e.g., naked singularities occur in Tolman-Bondi space-times where

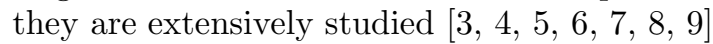

Comparatively, much less is known about singularity formation in higher dimensions (HD) that are being currently considered very important in view of recent developments in string theory and other field theories. In string and M-theory, which may provide a route towards quantum gravity, gravity is truly higher dimensional interaction, which becomes effective four dimensional (4D) at low energies. This provoked great interest among theoretical physicist in studying physics in HD [10]. It would be, therefore, highly desirable to understand the final fate of gravitational collapse in HD space-times. To understand the general collapse problem as well as nature of singularities, one would like to analyze exact solutions, preferably in close form, of HD Einstein equations. However the non-linearity of field equations makes them difficult, even in spherical symmetry.

A model in which analytical treatment appears feasible is that of 5D Tolman-Model that describes gravitational collapse of spherically symmetric inhomogeneous dust in a $5 \mathrm{D}$ space-time, since in this case the general exact solutions, for both marginally bound $(f=0)$ and non-marginally bound $(f \neq 0)$, are known in close form

\footnotetext{
*E-mail:sgghosh@iucaa.ernet.in

†E-mail:asitb@cal3.vsnl.net.in
}

11. This is unlike the $4 \mathrm{D}$ case, where the cooresponding solution is available in parametric form only. Hence, we shall restrict ourselves to the 5D non-marginally bound collapse. Further, the 5D space-time is particularly more relevant because both 10D and 11D supergravity theories yield solutions where a $5 \mathrm{D}$ space-time results after dimensional reduction [12].

The objective of this paper is to analyze the collapse of an inhomogeneous dust cloud in a 5D self-similar space-time and extend the earlier results [13, 14 beyond marginally bound case. We also access the curvature strength of central shell focusing singularities. We find that gravitational collapse of a self-similar 5D spacetime gives rise to a strong-curvature shell focusing naked singularity, providing an explicit counter-example to the CCC.

We have used units which fix the speed of light and the gravitational constant via $8 \pi G=c=1$.

\section{HIGHER DIMENSIONAL TOLMAN-BONDI MODELS}

We start with a brief summary of the 5D spherical symmetric inhomogeneous dust models. The metric for the $5 \mathrm{D}$ case, in comoving coordinates, assumes the form:

$$
d s^{2}=-e^{\nu(t, r)} d t^{2}+e^{\lambda(t, r)} d r^{2}+R^{2}(t, r) d \Omega^{2}
$$

where $d \Omega^{2}=d \theta_{1}^{2}+\sin ^{2} \theta_{1}\left(d \theta_{2}^{2}+\sin ^{2} \theta_{2} d \theta_{3}^{2}\right)$ is the metric of a 3 sphere, $r$ is the comoving radial coordinate, $t$ is the proper time of freely falling shells, $R$ is a function of $t$ and $r$ with $R \geq 0$ (equality holds at the origin) [14. Here

$$
\begin{aligned}
e^{\nu} & =1 \\
e^{\lambda} & =\frac{R^{\prime 2}}{1+f(r)}
\end{aligned}
$$

where the prime denotes a partial derivative with respect to $r, f(r)$ is an arbitrary function satisfying

$$
f(r)>-1
$$


and $R^{\prime}$ is assumed to be positive, in order to avoid situation where negative mass shells are present [16. The function $R(t, r)$ is the solution of

$$
\dot{R}^{2}=\frac{F(r)}{R^{2}}+f(r)
$$

where the over-dot denotes the partial derivative t. Since in the present discussion we are concerned with collapse, we require that $\dot{R}(t, r)<0$. The functions $F(r)$ is another arbitrary function and like $f(r)$, it also results from the integration of the field equations. The function $F$ must be positive, because $F<0$ implies the existence of negative mass. This can be seen from the mass function $m(t, r)$ 16], which in the 5D Tolman-Bondi case is given by

$$
m(t, r)=R^{2}\left(1-g^{a b} R,_{a} R, b\right)
$$

Using Eqs. (11) and (5), this implies that

$$
m(r)=F(r)
$$

Equation (5) has three types of solutions, namely, hyperbolic, parabolic and elliptic solutions, depending on whether $f(r)>0, f(r)=0$ or $f(r)<0$, respectively. We note that $F^{\prime}$ (as well as F) must be positive because mass-energy density $\epsilon(t, r)$, which is given by

$$
\epsilon(t, r)=\frac{3 F^{\prime}}{2 R^{3} R^{\prime}}
$$

must be non-negative, and since we have assumed $R^{\prime}>0$, it follows that $F^{\prime}>0$.

Integration Eq. (5) shows that the evolution of the shell is given by

$$
R^{2}=\left[f\left[t_{c}(r)-t\right]^{2}+2 \sqrt{F}\left[t_{c}(r)-t\right]\right]
$$

and where $t_{c}(r)$ is a function of integration which represents the time taken by the shell with coordinate $r$ to collapse to the center. This is a new exact solution of $5 \mathrm{D}$ spherical collapse found, recently, by Banerjee and Chatterjee 11. The solution (9) is not unique. In an earlier paper 14] a different solution was given. But, main problem with that solution is that one cannot smoothly pass over to marginally bound case when $f=0$. The solution (9) has no such disadvantage. Hence we have chosen the solution (9) to study nature of singularities for all cases: $f>0, f=0$ as well as $f<0$. The Eq. (5) is also interesting in the sense that in the analogous $4 \mathrm{D}$ case the solutions can't be obtain in close form [4, 7]. The three arbitrary functions $F(r), f(r)$ and $t_{c}(r)$ completely specify the behavior of shell of radius $r$.

It is possible to make an arbitrary relabelling of spherical dust shells by $r \rightarrow g(r)$, without loss of generality, we fix the labelling by requiring that, on the hypersurface $t=0, r$ coincides with the radius

$$
R(0, r)=r
$$

This corresponds to the following choice of $t_{c}(r)$

$$
t_{c}(r)= \begin{cases}-\frac{\sqrt{F} \mp \sqrt{F+f r^{2}}}{f}, & f \neq 0 \\ +\frac{r^{2}}{2 \sqrt{F}} & f=0\end{cases}
$$

The time coordinate and radial coordinate are respectively in the ranges $-\infty<t<t_{c}(r)$ and $0 \leq r<\infty$.

Let $\rho(r)$ be the function given by

$$
\rho(r) \equiv \epsilon(0, r)=\frac{3 F^{\prime}}{2 r^{3}} \Rightarrow F(r)=\frac{2}{3} \int \rho(r) r^{3} d r
$$

Given a regular initial surface, the time for the occurrence of the central shell-focusing singularity for the collapse developing from that surface is reduced as compared to the 5D case for the marginally bound collapse. The reason for this stems from the form of the mass function in Eq. (12). In a ball of radius 0 to $r$, for any given initial density profile $\rho(r)$, the total mass contained in the ball is greater than in the corresponding $5 \mathrm{D}$ case. In the 5D case, the mass function $F(r)$ involves the integral $\int \rho(r) r^{2} d r$ [2], as compared to the factor $r^{3}$ in the $5 \mathrm{D}$ case. Hence, there is relatively more mass-energy collapsing in the space-time as compared to the 5D case, because of the assumed overall positivity of mass-energy (energy condition). This explains why the collapse is faster in the 5D case.

In order to study the collapse of a finite spherical body, we have to match the solution along the time-like surface at some $r=r_{c}$ to the 5D Schwarzschild exterior. The 5D metric (11) can be matched to 5D Schwarzschild metric

$$
d s^{2}=-\left(1-\frac{m_{s}}{r_{s}^{2}}\right) d t^{2}+\left(1-\frac{m_{s}}{r_{s}^{2}}\right)^{-1} d r_{s}^{2}+r^{2} d \Omega^{2}
$$

on a spherical hypersurface $\Sigma$ and the junction conditions yield $F=m_{s}$ 11]. Here $r_{s}=R\left(t, r_{c}\right)$ and $m_{s}$ is the total mass enclosed within the coordinate radius $r_{c}$.

Further, 5D Friedmann solutions corresponds to $t_{c}(r)=0$, and with a suitable coordinate choice for $\mathrm{r}$ one can set $f(r)=-k r^{2}, R(t, r)=r S(t)$ and $F(r)=A r^{4}$ [11. Here $k$ and $A$ are constants, and $S(t)$ is the scale factor.

\section{EXISTENCE AND STRUCTURE OF NAKED SINGULARITIES}

It has been shown that Shell-crossing singularities (characterized by $R^{\prime}=0$ and $R>0$ ) are gravitationally weak and hence such singularities cannot be considered seriously. Hence, we shall confine our discussion to the central shell focusing singularity. The Kretschmann scalar ( $K=R_{a b c d} R^{a b c d}, R_{a b c d}$ the Riemann tensor). For the metric (11), it reduces to

$$
K=7 \frac{F^{\prime 2}}{R^{6} R^{\prime 2}}-36 \frac{F F^{\prime}}{R^{7} R^{\prime}}+72 \frac{F^{2}}{R^{8}}
$$


The Kretschmann scalar and energy density both diverge at $t=t_{c}(r)$ indicating the presence of a scalar polynomial curvature singularity [17]. It is known that, depending upon the inhomogeneity factor, the 5D Tolman-Bondi solutions admits a central shell focusing naked singularity in the sense that outgoing geodesics emanate from the singularity. Here we wish to investigate the similar situation in our $5 \mathrm{D}$ space-time.

The self-similar solutions have attracted considerable attraction in recent decades and they play a crucial role in many cosmological and astrophysical contexts 115. Spherically symmetric self-similar solutions of Einstein equations are characterized by the fact that the spacetime possess a homothetic killing vector. This means the solution is unchanged by transformation $t \rightarrow a t, r \rightarrow a r$ for any constant $a$. Thus the self-similarity demands that

$$
\begin{aligned}
& F(r)=\zeta^{2} r^{2} \\
& f(r)=\text { Const. } \\
& t_{c}(r)=B r
\end{aligned}
$$

The parameter $B$ gives the inhomogeneity of the collapse. For $B=0$ all shells collapse at the same time. For higher $B$ the outer shells collapse much later than the central shell. From Eqs. (11) and (17), we obtain an interesting relation

$$
\zeta=\frac{1-B^{2} f}{2 B}
$$

This expression reduces to $\zeta=1 / 2 B$ [13, 14] when $f=0$. We notice that $\zeta>0$, and so $B^{2} f<1$. We are interested in the causal structure of the space-time when the central shell collapses to the center $(R=0)$.

From Eqs. (12) and (15), the energy density at the singularity

$$
\rho=\frac{3 \zeta^{2}}{r^{2}}
$$

and the equation of general density becomes

$$
\begin{aligned}
& \epsilon=3 \zeta^{2} y /\left[t^{2}\left[f(B y-1)^{2}+2 \zeta y(B y-1)\right]\right. \\
& \times[f B(B y-1)+\zeta(2 B y-1)]]=\frac{C(y)}{t^{2}}
\end{aligned}
$$

where $y=t / r$ is usual similarity variable. Note that $\epsilon$ is singular at $t=B r$. The nature (a naked singularity or a black hole) of the singularity can be characterized by the existence of radial null geodesics emerging from the singularity. The singularity is at least locally naked if there exist such geodesics, and if no such geodesics exist, it is a black hole. The critical direction is the Cauchy horizon. This is the first outgoing null geodesic emanating from $r=t=0$. The Cauchy horizon of the self-similar space-time has $y=r / t=$ const [5, 6]. The equation for outgoing null geodesics is

$$
\frac{d t}{d r}=\frac{R^{\prime}}{\sqrt{1+f}}
$$

Hence along the Cauchy horizon, we have

$$
y^{2}{R^{\prime}}^{2}=1+f
$$

and using Eqs. (22) and (9), with our choice of the scale, we obtain the following algebraic equation:

$$
\begin{gathered}
(1+f)\left[f(B y-1)^{2}+2 \zeta y(B y-1)\right] \\
-y^{2}[f B(B y-1)+\zeta(2 B y-1)]^{2}=0
\end{gathered}
$$

This algebraic equation governs the behavior of the tangent vector near the singular point. The central shell focusing singularity is at least locally naked, if Eq. (23) admits one or more positive real roots. Hence in the absence of positive real roots, the collapse will always lead to a black hole. Thus, the occurrence of positive real roots implies that the strong $\mathrm{CCC}$ is violated, though not necessarily the weak CCC. If Eq. (23) has only one positive root, a single radial null geodesic would escape from the singularity, which amounts to a single wave front being emitted from the singularity and hence singularity would appear to be naked only, for an instant, to an asymptotic observer. A naked singularity forming in gravitational collapse could be physically significant if it is visible for a finite period of time, to an asymptotic observer, i.e., a family of geodesics must escape from the singularity. This happens only when Eq. 223) admits at least two positive real roots [2]. In this paper we are concerned only with such singularities. Hence we shall look for conditions for which Eq. (23) admits at least two positive real roots.

We start from the marginally bound case, which has been analyzed earlier [13, 14. We already know what happens and we refer the reader to these papers for details. Setting $f=0$, Eq. (23) simplifies to

$$
y \zeta^{2}(2 B y-1)^{2}=2 \zeta(B y-1)
$$

To facilitate comparison with the work of Ghosh and Beesham [13], we introduce a new variable $y=1 / X$ and use the value of $\zeta$ and after some rearrangement, Eq. (24) takes the same form as in [13]:

$$
X^{2}\left[1-\frac{X}{B}\right]=\left[1-\frac{1}{2 B} X\right]^{2}
$$

It can be shown that Eq. 25) has two positive roots if $B>B_{c}=1.6651$. This is slightly higher than the analogous value, $B_{c}^{4}=1.56736$, in $5 \mathrm{D}$. The corresponding Cauchy horizon evolves as $y=0.78611$. Thus in $5 \mathrm{D}$ one needs higher inhomogeneity to produce naked singularity. For $B>B_{c}$, two solutions exist, the largest $y$ gives the Cauchy horizon. Other solution is termed as self-similar horizon [6] (see Table I).

It is seen that Eq. (23) admit two positive roots for $y$ when $\zeta=\zeta_{c} \leq 0.30028$ and hence referring to our above discussion singularities are naked for $\zeta \in\left(0, \zeta_{c}\right.$ and black holes form otherwise. Thus $\zeta_{c}$ is the value of $\zeta$ where a transition from naked singularities to black holes 
TABLE I: Variation of $y, \lambda$ for different $B(f=0)$

\begin{tabular}{cccc}
\hline \hline$B$ & $\lambda$ & \multicolumn{2}{c}{$y$} \\
\hline 1.7 & 0.294118 & 0.714512, & 0.846397 \\
2 & 0.25 & 0.549139, & 0.929956 \\
2.5 & 0.2 & 0.420428, & 0.965243 \\
3 & 0.166667 & 0.344179, & 0.978711 \\
3.5 & 0.142857 & 0.292235, & 0.985525 \\
\hline \hline
\end{tabular}

occur. The quantity $\zeta_{c}$ is called critical parameter. It is interesting to see that the value of $\zeta_{c}$ decreases in $5 \mathrm{D}$ and hence one can say that naked singularity spectrum, of the $4 \mathrm{D}$ dust collapse, gets partially covered in $5 \mathrm{D}$. Our results are in agreement with earlier results. A similar situation also occur in higher dimensional radiation collapse 18].

Next we compare the behavior found in the marginally bound $(f=0)$ case with that found in non-marginally bound case. In order to investigate the changes introduced, at least qualitatively in the above picture when $f \neq 0$, we have solved (23) numerically and summarize the results in table II. For negative values of $f$, there is critical value, $B_{c}$, such that for $B \geq B_{c}$ collapse always leads to a visible singularity and covered otherwise. The numerical results show that for larger (smaller) positive curvature, i.e., for larger(smaller) magnitude of $f$ one needs a collapse with higher (lower) inhomogeneity to produce a naked singularity.

Stars are bound objects but an unbound state can occur, e.g., if there is collision of two stars. If $f>0$, we observe that collapse lead to a visible singularity for $B_{c} \leq B \leq B_{u}$, where $B_{u}$ is some upper bound for $B$. In this case, there are three solutions (see Table II). Further, it is interesting to note that $\zeta$ and $B_{C}$ decreases as we increase the value of $f$. Thus for larger (smaller) negative curvature one needs a collapse with lower (higher) inhomogeneity to produce a naked singularity.

Finally, the apparent horizon is formed when the boundary of trapped three spheres are formed. The apparent horizon is the solution of

$$
g^{a b} R_{, a} R_{, b}=\dot{R}^{2}-\frac{R^{\prime 2}}{e^{\lambda}}
$$

Considering Eqs. (3) and (5), we have the apparent horizon in the interior dust ball lies at $R^{2}=F$. The corresponding time $t_{\mathrm{ah}}(r)$ is given by

$$
t_{\mathrm{ah}}(r)=t_{c}(r)-\frac{1}{2} \sqrt{F}
$$

and because of Eqs. (17), (11) and (27): $t_{\mathrm{ah}}(r)-$ ${ }^{t}$ Cauchy $(r)<0$. Thus apparent horizon always appear before the Cauchy horizon has formed and singularity is globally naked. The global nakedness of the singularity can then be seen by making a junction onto 5D Schwarzschild space-time [5, 6].
TABLE II: Variation of $B_{C} \lambda$ and $y_{0}$ for various $f$

\begin{tabular}{cccc}
\hline \hline$f$ & $B_{C}$ & $\lambda$ & Equal roots $y_{0}$ \\
\hline-0.4 & 4.16347 & 0.952787 & 0.3978 \\
-0.3 & 2.82096 & 0.600388 & 0.53331 \\
-0.2 & 2.23607 & 0.447214 & 0.63245 \\
0 & 1.6651 & 0.300283 & 0.78611 \\
0.4 & 1.18805 & 0.183246 & 1.01733 \\
1 & 0.890515 & 0.116216 & 1.28286 \\
2 & 0.671855 & 0.0723525 & 1.62888 \\
3 & 0.560097 & 0.0525573 & 1.91208 \\
\hline \hline
\end{tabular}

\section{A. Strength of Naked Singularity}

An important aspect of a singularity is its gravitational strength [19]. There have been attempts to relate it to stability [9]. A singularity is gravitationally strong or simply strong if it destroys by crushing or stretching any object which fall into it. It is widely believed that a space-time does not admit an extension through a singularity if it is a strong curvature singularity in the sense of Tipler [20]. Clarke and Królak [21] have shown that a sufficient condition for a strong curvature singularity as defined by Tipler [20] is that for at least one non-space like geodesic with affine parameter $k$, in the limiting approach to the singularity, we must have

$$
\lim _{k \rightarrow 0} k^{2} \psi=\lim _{k \rightarrow 0} k^{2} R_{a b} K^{a} K^{b}>0
$$

where $R_{a b}$ is the Ricci tensor. Our purpose here is to investigate the above condition along future directed radial null geodesics, which emanate from the naked singularity. Now $k^{2} \psi$, with the help of Einstein equations and Eq. (20) can be expressed as

$$
k^{2} \psi=C\left[\frac{k}{t} \frac{d t}{d k}\right]^{2}
$$

Clearly the null geodesic equations for Cauchy horizon give $t=k^{\alpha}$ 河. We find that

$$
\lim _{k \rightarrow 0} k^{2} \psi=C_{0} \alpha^{2}>0
$$

where $\lim C=C_{0}$. Thus, along Cauchy horizon, the strong curvature condition is satisfied.

\section{CONCLUDING REMARKS}

The Tolman-Bondi metric has been widely used, in 4D case, to understand the final fate of gravitational collapse. It has been found that both naked singularities and black holes form depending upon the choice of initial data. Indeed, one can safely assert that end state of 4D Tolman-Bondi collapse is now completely known in dependence of choice of initial data. In the absence of the proof of either version of CCC, it was used as 
tool to get insights in to more general gravitational collapse situations. In this paper we have investigated the influence of extra dimensions on Tolman-Bondi dust collapse. The occurrence and curvature strength of a shell focusing naked singularity in a 5D self-similar spherically symmetric collapse of a dust cloud has been investigated. We found that the scenario changes in many ways. The extra dimensions is a shrinkage of the naked singularity initial data space, or an enlargement of the black hole initial data space of 4D Tolman-Bondi collapse. We found that naked singularities in our case develop for a slightly higher value of the inhomogeneity parameter in comparison to the analogous situation in the $4 \mathrm{D}$ case. Thus the presence of an extra dimension does alter the established picture of 4D Tolman-Bondi collapse, but cannot completely cover the naked singularities. Further, Along the null ray emanating from the naked singularity, the strong curvature condition (28) is satisfied. The formation of these naked singularities violates the CCC.

The crucial issue associated with naked singularities is the question of their stability. The main point is that if naked singularities are not stable or generic in some suitable sense, then they may not be physically realizable. However, the problem is that we do not have well defined criteria of stability. Under such circumstances, one can consider a perturbation of currently available collapse scenarios and examine if the singularity still persists or gets covered. It is worth noting that, by introduction of extra dimensions, there exist a set of nonzero measure of values of $\zeta$ for which strong curvature naked singularities develop, and the CCC is violated. Thus presence of extra dimensions do not remove naked singularity of $4 \mathrm{D}$ Tolman-Bondi collapse. As a result, one can say that the naked singularity of 4D Tolman-Bondi collapse is stable to the introduction extra dimension.

The exact analysis discussed, for non-marginally bound case, is specialized for the 5D Tolman-Bondi models and can't be done even in analogous $4 \mathrm{D}$ case.

In conclusion, we have studied the development of a strong curvature naked singularity in 5D Tolman-Bondi collapse that violates the strong CCC. The Kretschmann scalar diverges in the approach to the singularity. Thus the naked singularity in 5D Tolman-Bondi collapse could be considered as a physically significant curvature singularity.

\section{Acknowledgments}

Authors would like to thank IUCAA, Pune for a visit under a ssociate-ship programme where this work was done.
[1] R. Penrose, Riv. Nuovo Cimento 1, 252 (1969); in General Relativity, an Einstein Centenary Volume, ed. S. W.Hawking and W. Israel (Cambridge University Press, Cambridge, England, 1979).

[2] P. S. Joshi, Global Aspects in Gravitation and Cosmology (Clendron, Oxford, 1993); C.J.S. Clarke, Class. Quantum Grav. 10, 1375 (1993); R.M. Wald, gr-qc/9710068; S. Jhingan and G. Magli, gr-qc/9903103; T.P. Singh, J. Astrophys. Astron. 20, 221 (1999); P.S. Joshi, Pramana 55, 529 (2000).

[3] D.M. Eardley and L. Smarr, Phys. Rev. D 19, 2239 (1979); D.M. Eardley, Gravitation in Astrophysics, ASI Series, edited by B. Carter and J.B. Hartle (NATO Advanced Study Institute, Series B: Physics, Vol 156)(Plenum Press, New York, 1986) pp 229-235.

[4] R.P.A.C. Newman, Class. Quantum Grav. 3, 527 (1986).

[5] B. Waugh and K. Lake, Phys. Rev. D 38, 1315 (1988).

[6] J.P.S. Lemos, Phys. Lett. A 158, 271 (1991); Phys. Rev. Lett. 68, 1447 (1992).

[7] I.H. Dwivedi and P.S. Joshi, Class. Quantum Grav. 9, L69 (1992). P.S. Joshi and T.P. Singh, Gen. Relativ. Gravit. 27, 921 (1995); Phys. Rev. D 51, 6778 (1995).

[8] P.S. Joshi and I.H. Dwivedi, Phys. Rev. D 47, 5357 (1993).

[9] S.S. Deshingkar, P.S. Joshi, and I.H. Dwivedi, Phys. Rev. D 59, 044018 (1999).

[10] J. Soda and K. Hirata, Phys. Lett. B 387, 271 (1996);
A. Ilha and J.P.S. Lemos, Phys. Rev. D 55, 1788 (1997); A. Ilha, A. Kleber and J.P.S. Lemos, J. Math. Phys. 40, 3509 (1999); A. V. Frolov Class. Quantum Grav. 16, 407 (1999); J.F.V. Rocha and A. Wang ibid. 17, 2589 (2000).

[11] A. Banerjee and S. Chatterjee, Pre-print (2002).

[12] J.J. Schwarz, Nucl. Phys. B226, 269 (1983).

[13] S.G. Ghosh and A. Beesham Phys. Rev. D 64, 124005 (2001).

[14] A. Banerjee, A. Sil and S. Chatterjee Astrophys. J. 422, 681 (1994); A. Sil and S. Chatterjee Gen. Relativ. Gravit. 26, 999 (1994).

[15] B.J. Carr and A.A. Coley, Class. Quantum Grav. 16, R31 (1999).

[16] C.W. Misner and D. Sharp Phys. Rev. 136, b571 (1964).

[17] S.W. Hawking and G.F.R. Ellis, The Large Scale Structure of Space-time (Cambridge University Press, Cambridge, 1973).

[18] S.G. Ghosh and N. Dadhich Phys. Rev. D 64, 047501 (2001).

[19] F.J. Tipler Phys. Lett. A 64,8 (1987).

[20] F.J. Tipler, C.J.S. Clarke, and G.F.R. Ellis in General Relativity and Gravitation, edited by A Held (Plenum, New York, 1980).

[21] C.J.S. Clarke and A. Królak J. Geom. Phys. 2, 127 (1986). 